\title{
Second harmonic generation in monolithic lithium niobate metasurfaces
}

\author{
Luca Carletti, ${ }^{1,2,3}$ (1) Cheng LI, ${ }^{1,4}$ (i) Jürgen Sautter, ${ }^{5}$ \\ Isabelle Staude, ${ }^{5}$ Costantino De Angelis, ${ }^{2}$ (i) TAO LI, ${ }^{4}$ (I) AND \\ Dragomir N. NeSheV $1{ }^{1, *}$ \\ ${ }^{1}$ Nonlinear Physics Centre, Research School of Physics, The Australian National University, Canberra, \\ ACT 2601, Australia \\ ${ }^{2}$ Department of Information Engineering, University of Brescia and INO-CNR, Via Branze 38, Brescia \\ 25123, Italy \\ ${ }^{3}$ Department of Information Engineering, University of Padova, Via Gradenigo 6/a, Padova 35131, Italy \\ ${ }^{4}$ College of Engineering and Applied Sciences, Nanjing University, Nanjing 210093, China \\ ${ }^{5}$ Institute of Applied Physics, Abbe Center of Photonics, Friedrich Schiller University Jena, 07745 Jena, \\ Germany \\ *Dragomir.Neshev@anu.edu.au
}

\begin{abstract}
Second-order nonlinear metasurfaces have proven their ability to efficiently convert the frequency of incident signals over subwavelength thickness. However, the availability of second-order nonlinear materials for such metasurfaces has so far been limited to III-V semiconductors, which have low transparency in the visible and impose constraints on the excitation geometries due to the lack of diagonal second-order susceptibility components. Here we propose a new design concept for second-order nonlinear metasurfaces on a monolithic substrate, which is not limited by the availability of thin crystalline films and can be applied to any non-centrosymmetric material. We exemplify this concept in a monolithic Lithium Niobate metasurface with cylinder-shaped corrugations for enhanced field confinement. By optimizing the geometrical parameters, we show enhanced second harmonic generation from a near-infrared pump beam with conversion efficiency above $10^{-5}$ using $1 \mathrm{GW} / \mathrm{cm}^{2}$ pump intensity. Our approach enables new opportunities for practical designs of generic metasurfaces for nonlinear and quantum light sources.
\end{abstract}

(C) 2019 Optical Society of America under the terms of the OSA Open Access Publishing Agreement

\section{Introduction}

Second harmonic generation (SHG) is one of the most studied nonlinear optical effects due to its huge range of potential applications, such as laser sources and microscopy [1]. However, since the optical nonlinearity of natural materials is intrinsically low, different techniques to enhance the nonlinear process have been proposed. A successful approach for the enhancement of SHG in photonic structures whose dimensions range from hundreds of micrometers up to a few millimeters relies on the phase-matching condition [1]. Among the different materials for fabrication of such nonlinear structures, lithium niobate $\left(\mathrm{LiNbO}_{3}\right)$ is one of the most commonly used due to its high second-order nonlinear response [2-4].

In the last two decades, the use of resonant photonic nanostructures has been successfully applied for enhancing SHG [5-8]. By inducing strong electromagnetic resonances, the electric field intensity is locally enhanced, as compared to unstructured materials. The local field enhancement is derived by the quality factors of the resonances at the participating wavelengths, the overlap of the photonic modes and their coupling to free space. The strength of this approach is that phase-matching is no longer required to promote SHG, while the nonlinear phenomena are boosted by several orders of magnitudes when the resonances of the cavity coincide with the fundamental and $\mathrm{SH}$ frequencies. 
In this context, plasmonic resonators have emerged as a viable choice [8], however, the high Ohmic loss of metals has limited their practical use. In recent years, several works have demonstrated the potential for nonlinear enhancement in high-refractive-index dielectric nanoparticles thanks to their negligible absorption loss and high-quality factor resonances [5,7]. For instance, AlGaAs nanodisks demonstrated SHG with a conversion efficiency of up to $0.01 \%$ [9]. However, the symmetry of the nonlinear second-order susceptibility tensor associated with $\mathrm{AlGaAs}$ (and in general of crystals with zincblende structure) results in a complex structure of the generated SH light [6,9,10-15]. Due to the off-diagonal nature of the quadratic susceptibility tensor of these materials, the SH emission normal to the metasurface is usually prohibited, which imposes further constraints on their applicability. Recent works have attempted to resolve this issue by beam-inclination [10] or by employing III-V semiconductors grown in the (111) crystalline direction [16,17], however, the success has been limited.

Alternative materials, such as $\mathrm{LiNbO}_{3}$ and other ferroelectrics, exhibiting strong diagonal second-order nonlinear susceptibility tensor, may open new avenues to nonlinearly light generation in dielectric metasurfaces. The main obstacles in using such material platforms, however, are the challenges of growth of thin crystalline films on low refractive index substrates. Furthermore, the subsequent nanostructuring of such materials represents another major challenge. The need of nanostructures on a low-refractive-index substrate is perceived as a necessary condition for supporting Mie-type resonance. While significant progress has been recently made in the development of $\mathrm{LiNbO}_{3}$ micro and nanostructures [18-20], including nanoresonators and metasurfaces [21-23], they rely on high-cost wafer bonding techniques. Therefore, a universal solution for quadratic nonlinear metasurfaces that does not depend on crystalline thin films is highly demanded.

Here, for the first time, we propose $\mathrm{LiNbO}_{3}$-on- $\mathrm{LiNbO}_{3}$ (monolithic) metasurfaces of z-cut $\mathrm{LiNbO}_{3}$ material, structured with nanopillar array for SHG. We demonstrate that such nanopillar array can exhibit a strong in-plane magnetic dipole (MD), resonant at wavelengths between 800 $\mathrm{nm}$ and $1000 \mathrm{~nm}$, despite the lack of low-index substrate. Our numerical nonlinear simulations show an enhancement of the SHG conversion efficiency, exceeding $5 \times 10^{-5}$ when illuminating the metasurface with a plane wave of an intensity of $1 \mathrm{GW} / \mathrm{cm}^{2}$.

Our design is comparable to the designs of monolithic Si-based high-contrast gratings, providing enhanced reflectivity for VCSELs [24,25], light-trapping for photovoltaic structures [26], and suppressed scattering for antireflection coatings [27]. However, the use of such gratings with ferroelectric materials for enhancement of the nonlinear processes has not been studied. While SHG in single disk-shaped corrugated GaP-on-GaP structures has been recently observed [28], the refractive index of ferroelectric materials, such as $\mathrm{LiNbO}_{3}$ in the visible spectral range is much smaller than the one of $\mathrm{GaP}$ or $\mathrm{Si}$. The lower refractive index of ferroelectric materials inevitably impacts on the optical field confinement and resonant phenomena. Therefore, the investigation of the linear and nonlinear monolithic metasurfaces represents a valuable and important challenge to assess the potential of such monolithic metasurface platform.

\section{Linear analysis}

A schematic representation of SHG in a monolithic $\mathrm{LiNbO}_{3}$ metasurface is shown in Fig. 1(a). The incident field is a plane wave propagating along the normal direction (i.e. z-axis). It illuminates the metasurface from the backside with an electric field that is linearly polarized, parallel to the $y$-axis. The geometrical parameters defining the unit cell of the metasurface are shown in Fig. 1(b). The nanoantennas are shaped as cylindrical pillars with a radius $r$ and a height $\mathrm{h}$. The distance between the centers of two neighboring nanoantennas represents the array's pitch p. Full-vectorial numerical simulations of the periodic structures are performed on a single array unit cell using periodic boundary conditions on the sidewalls of the simulation domain (i.e. orthogonal to the array) satisfying Floquet condition with a phase of 0 radians to work at 
normal incidence. The simulations account for the dispersion and birefringence of the $\mathrm{LiNbO}_{3}$ permittivity using the Sellmeier model [29]. We focus on the design of monolithic metasurfaces in a $\mathrm{z}$-cut $\mathrm{LiNbO}_{3}$ wafer (i.e. anisotropy axis parallel to the z-axis), which is potentially easier for nanostructuring [30]. In this geometry, we expect SHG enhancement only due to the optical resonances of the nanostructures since such z-cut design does not allow for SH phase matching in the bulk $\mathrm{LiNbO}_{3}$ substrate.

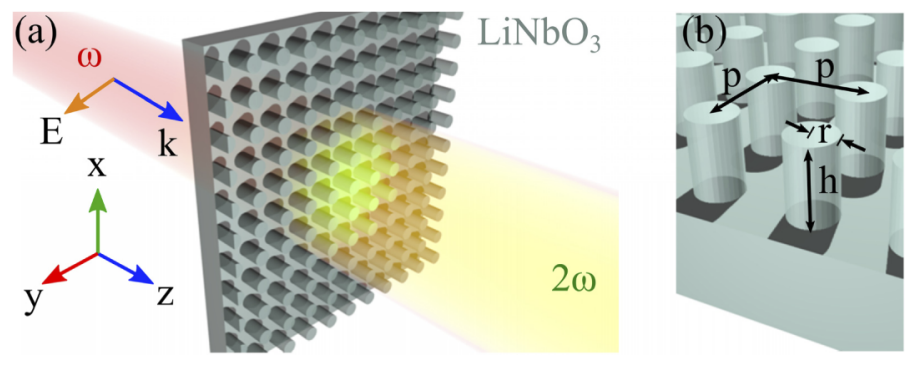

Fig. 1. (a) Schematic design of the monolithic metasurface for second harmonic generation. Incident plane wave is polarized along the y-axis. The pump beam direction and electric field are indicated in terms of $k$ and $E$. (b) Schematic of the metasurface unit cell. The pillar-shaped nanoantenna has a radius $r$ and a height $h$. The pitch of the squire unit cell is $p$.

We first study the linear response of the array in the visible and near-infrared wavelength range between $400 \mathrm{~nm}$ and $1000 \mathrm{~nm}$. Figure 2(a) shows the reflectivity spectrum of the metasurface made of pillars with $\mathrm{r}=150 \mathrm{~nm}, \mathrm{~h}=440 \mathrm{~nm}$, and a pitch $\mathrm{p}=590 \mathrm{~nm}$. The spectrum exhibits several local maxima and minima of the reflectivity for wavelengths shorter than $800 \mathrm{~nm}$ and a low reflectivity plateau for wavelengths above $800 \mathrm{~nm}$. To understand the nature of this complex optical response, we perform a multipolar analysis of the electric field induced in the nanopillars [31], as shown in Fig. 2(a) right axis. We can observe a pronounced MD resonance at a wavelength of $815 \mathrm{~nm}$. As shown in Fig. 2(b), at this wavelength, the electric field inside the nanopillar circulates in the yz plane through the axis of the cylinder, resembling an in-plane MD. At the resonance condition, the maximum field intensity enhancement (FE) defined as the ratio $\left(|\mathrm{E}| / \mathrm{E}_{0}\right)^{2}$, where $\mathrm{E}$ is the amplitude of the electric field and $\mathrm{E}_{0}$ is the amplitude of the incident plane wave, is over 40. This value is comparable to single AlGaAs disks in air [12] or on low refractive index substrates [9,11]. At wavelengths shorter than $800 \mathrm{~nm}$, we can also observe higher-order resonances such as electric dipole (ED) and electric quadrupole (EQ) at about 650 $\mathrm{nm}$, and a magnetic quadrupole (MQ) at about $425 \mathrm{~nm}$. We note that $\mathrm{LiNbO}_{3}$ is transparent for wavelengths between 350 and $5200 \mathrm{~nm}$. Our analysis shows that the monolithic $\mathrm{LiNbO}_{3}$ array can support resonances over a wide wavelength range, covering both the pump (800-1000 nm) and second harmonic (400-500 nm) wavelengths.

To understand the dependence of the electric field enhancement on the unit cell geometrical parameters, we define the average E-field intensity enhancement, $\mathrm{FE}_{\mathrm{avg}}$, as

$$
F E_{a v g}=\frac{\iiint F E d V}{V}=\frac{\iiint\left(E / E_{0}\right)^{2} d V}{V},
$$

where the integration volume is inside the entire nanoantennas, V. As can be seen in Fig. 3(a), the average E-field intensity enhancement peak occurs in correspondence of the MD resonant peak. As the pillar radius is increased, its spectral position shifts towards longer wavelengths. Figure 3(b) shows the $\mathrm{FE}_{\text {avg }}$ spectra, as a function of cell pitch. We can observe that the FE peak slowly shifts towards longer wavelengths, as the pitch is increased. This weaker dependence of the MD mode on the lattice periodicity indicates that the resonance is mainly defined by the 


\section{Optics EXPRESS}
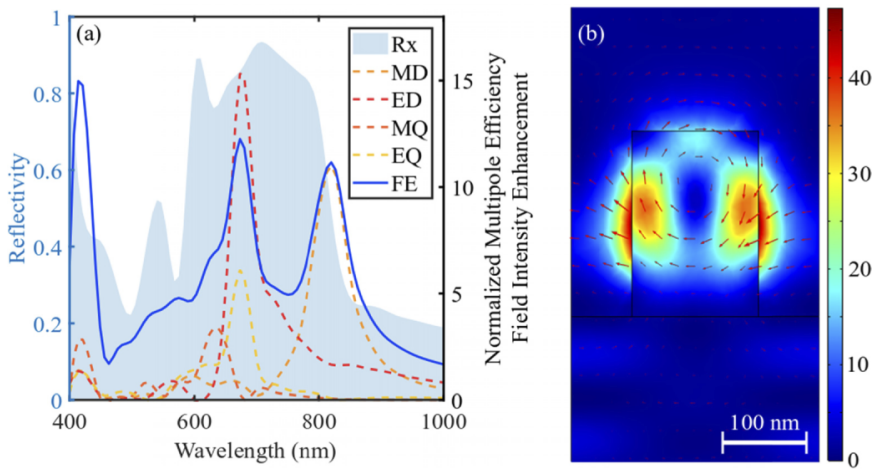

Fig. 2. (a) Reflectivity and multipolar analysis of the fields generated in the nanopillar as a function of wavelength, calculated for $\mathrm{r}=150 \mathrm{~nm}, \mathrm{p}=590 \mathrm{~nm}$ and $\mathrm{h}=440 \mathrm{~nm}$. Rx stands for reflectivity, MD for magnetic dipole, ED for electric dipole, MQ for magnetic quadrupole, EQ for electric quadrupole, and FE for field intensity enhancement. (b) Cross-section of the electric field intensity enhancement $\left(|\mathrm{E}| / \mathrm{E}_{0}\right)^{2}$ in the yz plane through the axis of the cylinder at a wavelength of $815 \mathrm{~nm}$. $\mathrm{E}_{0}$ is the amplitude of the incident plane wave. The arrows represent the E-field vector components in the same yz plane.

properties of the individual nanostructures. Figure 3(c) reports the $\mathrm{FE}_{\text {avg }}$ spectra as a function of the pillar height. We can observe that the $\mathrm{FE}_{\text {avg }}$ peak wavelength is nearly constant with respect to the pillar height, suggesting a low impact on the MD resonance. These results show that even with the lack of a low-permittivity substrate, subwavelength dielectric nanoresonators can be defined by corrugating the surface of the material. Importantly, such resonators can exhibit magnetic and electric Mie-type resonances with appreciable field enhancement factors. In the following, we show that by finely adjusting the design parameters and incidence polarization, we can engineer such resonances to dramatically enhance the SHG efficiency.
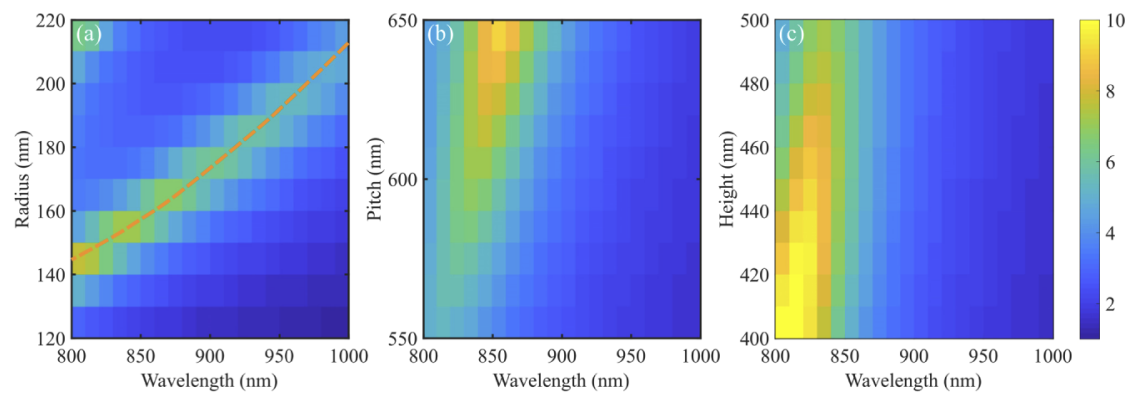

Fig. 3. Average E-field intensity enhancement spectra as a function of (a) pillar radius $(\mathrm{p}=600 \mathrm{~nm}, \mathrm{~h}=500 \mathrm{~nm}),(\mathrm{b})$ metasurface pitch $(\mathrm{r}=150 \mathrm{~nm}, \mathrm{~h}=500 \mathrm{~nm})$, and (c) pillar height $(\mathrm{p}=590 \mathrm{~nm}, \mathrm{r}=150 \mathrm{~nm})$. The dashed line on (a) shows the MD peak as a function of radius and wavelength.

\section{Second harmonic generation in monolithic metasurfaces}

Next, we study the process of SHG in such monolithic metasurfaces using finite-element-method numerical simulations in COMSOL in the undepleted pump approximation. Thus, we use two subsequent simulations: at the first step, the linear optical response of the metasurface at the fundamental wavelength (FW) is evaluated; at the second step, we reproduce the second harmonic 
(SH) response by excitation of the metasurface with the nonlinear currents induced by the FW beam [12]. These are calculated considering the $\mathrm{LiNbO}_{3}$ nonlinear coefficient matrix:

$$
\left[\begin{array}{cccccc}
0 & 0 & 0 & 0 & d_{31} & -d_{22} \\
-d_{22} & d_{22} & 0 & d_{31} & 0 & 0 \\
d_{31} & d_{31} & d_{33} & 0 & 0 & 0
\end{array}\right],
$$

where $\mathrm{d}_{22}=2.1 \mathrm{pm} / \mathrm{V}, \mathrm{d}_{31}=-4.6 \mathrm{pm} / \mathrm{V}, \mathrm{d}_{33}=-25.2 \mathrm{pm} / \mathrm{V}$, extracted from Ref. [1]. We define the SH conversion efficiency, $\eta$, as

$$
\eta=\frac{P^{2 \omega}}{P_{\text {in }}}=\frac{\int(\mathbf{S} \cdot \hat{\mathbf{n}}) d A}{I_{\text {in }} \cdot p^{2}},
$$

where $\mathbf{S}$ is the Poynting vector of the $\mathbf{S H}$ field, $\hat{\mathbf{n}}$ is the unit vector normal to the integration surface, which covers the top and bottom of the array, and $I_{\text {in }}$ is the incident intensity.

The dependences of SHG efficiency on the geometrical parameters of the metasurface are shown in Fig. 4. To improve the SHG efficiency, we vary the three geometrical parameters (radius, pitch, and height) recursively; each time before we start varying the next parameter, we fix the scanned parameter(s) at its/their best value. We chose a starting point at $\mathrm{p}=600$ $\mathrm{nm}$ and $\mathrm{h}=500 \mathrm{~nm}$. First, we study the SHG conversion efficiency spectrum as a function of the nanopillar radius, Fig. 4(a). We can deduce that the SH peak corresponds to dimensions accounting for the MD resonance at the FW (see Fig. 2). The increase of the pillar radius red-shifts the SHG efficiency peak, as expected due to the analog shift of the MD resonance and the associated $E$-field intensity enhancement, see Fig. 3(a) [12]. Slight differences in the spectral position of the peaks of E-field intensity enhancement and SH conversion efficiency might be due to mode selection rules at the $\mathrm{SH}$ wavelength.
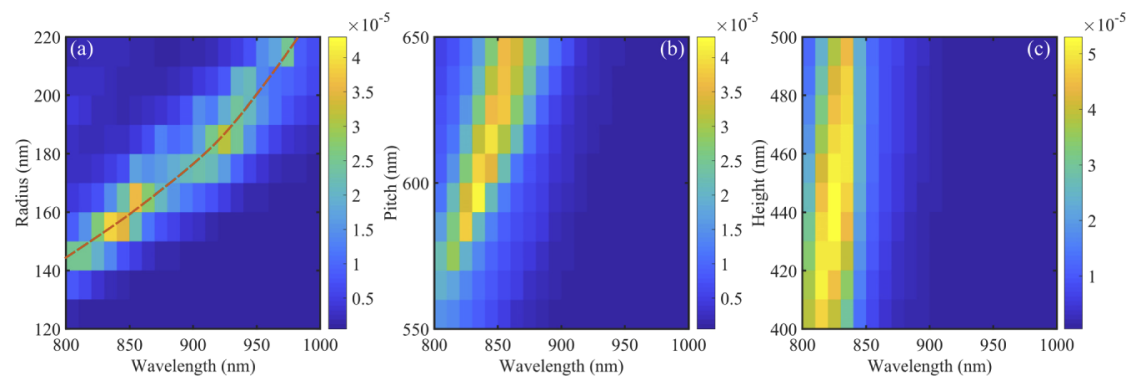

Fig. 4. Total SH conversion efficiency as a function of the pump wavelength and (a) pillar radius $(\mathrm{p}=600 \mathrm{~nm}, \mathrm{~h}=500 \mathrm{~nm})$, (b) array pitch $(\mathrm{r}=150 \mathrm{~nm}, \mathrm{~h}=500 \mathrm{~nm})$, and (c) pillar height $(\mathrm{r}=150 \mathrm{~nm}, \mathrm{p}=590 \mathrm{~nm})$. The dashed line on (a) shows the MD peak as a function of radius and wavelength.

The second parameter that we study is the metasurface pitch, as shown in Fig. 4(b). We focus on the case of a sub-diffraction pitch at the FW by varying $p$ between 550 and $650 \mathrm{~nm}$. The firsts diffraction orders, however, are present at the SH wavelength since $p>\lambda_{\mathrm{SH}}$. We can observe that the SH conversion efficiency exhibits a peak in the area where the $E$-field intensity enhancement at the FW is also maximized, see Fig. 3(b). However, the SH peak is narrower than the one in Fig. 3(b) due to mode selection at the SH wavelength. At last, we characterize the $\mathrm{SH}$ efficiency spectra as a function of the pillar height from 400 to $500 \mathrm{~nm}$. The upper limit is chosen to maintain a subwavelength height of the $\mathrm{LiNbO}_{3}$ pillar. As can be seen in Fig. 4(c), the SHG peak is obtained for the optimum pillar height of $440 \mathrm{~nm}$. This is likely due to the matching 
between phase retardation effect in the nanopillar between the top and bottom surfaces and the nanopillar height.

From this analysis we find that arrays composed of nanopillars with $r=150 \mathrm{~nm}, h=440 \mathrm{~nm}$ and with $p=590 \mathrm{~nm}$ can yield to a maximum SHG efficiency, exceeding $5 \times 10^{-5}$ when the FW is at $815 \mathrm{~nm}$. For the geometrical parameters space explored in this study, we can observe a strong correlation between the nonlinear efficiency and the pillar radius: even if the radius changes just a little, the nonlinear response of similar value can no longer be observed at the same wavelength. In contrast, for different pitch and height, the changes in the SH efficiency is apparently weaker. However, if a different parametric space was explored the effects of pitch and pillar height might increase. This agrees well with the dispersion of the average $E$-field intensity enhancement vs. the geometrical parameters observed in Fig. 3 and suggests a weak coupling between the adjacent $\mathrm{LiNbO}_{3}$ pillars.

To understand the contribution from the $\mathrm{LiNbO}_{3}$ substrate, we perform numerical simulations where the nonlinear currents are generated in the substrate only. The thickness of the $\mathrm{LiNbO}_{3}$ in the simulations is chosen to be equal to the coherence length, $\mathrm{L}_{\mathrm{c}}$, for SHG from FW of $815 \mathrm{~nm}$, where $\mathrm{L}_{\mathrm{c}}=\lambda_{\mathrm{FW}} / 4\left(\mathrm{n}_{\mathrm{FW}}-\mathrm{n}_{\mathrm{SH}}\right)=1.17 \mu \mathrm{m}$ with $\mathrm{n}_{\mathrm{FW}}$ and $\mathrm{n}_{\mathrm{SH}}$ the refractive indices at $\mathrm{FW}$ and $\mathrm{SH}$ respectively [1]. These simulations show that the SH conversion efficiency from the substrate is about $3 \times 10^{-7}$ which is two orders of magnitude smaller than when the nonlinear currents are generated in the nanopillars. This result confirms that the SHG is enhanced mostly by the metasurface optical resonant response.

Considering that in the analyzed wavelength range the dispersion of the permittivity of $\mathrm{LiNbO}_{3}$ is low, we can exploit the scalability of Maxwell's equations to tune our design for pump wavelengths from $825 \mathrm{~nm}$ up to $1000 \mathrm{~nm}$. By fixing the ratio between radius, pitch, and height at the optimum (i.e. $\mathrm{r}: \mathrm{p}: \mathrm{h}=150: 590: 440 \mathrm{~nm}$ ) and simultaneously scaling the three geometric parameters, we can tune the FW for which the maximum SHG is achieved, as shown in Fig. 5(a). Note that such scaling enables the nonlinear generation of light from $400 \mathrm{~nm}$ to $500 \mathrm{~nm}$ with nearly constant SH conversion efficiency. The enhancement of the SHG in the optimized structures is analyzed as a function of the incident E-field polarization.
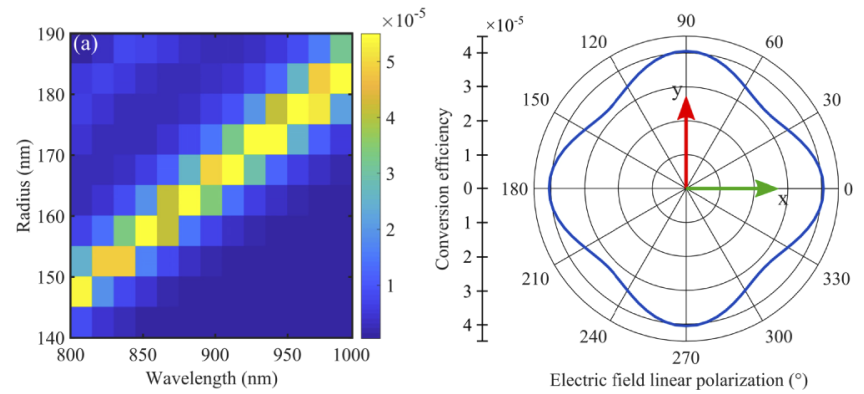

Fig. 5. (a) Total SH conversion efficiency as a function of the pump wavelength and geometry for a fixed ratio between radius, pitch, and height of $150 \mathrm{~nm}: 590 \mathrm{~nm}$ : $440 \mathrm{~nm}$. (b) SH conversion efficiency for FW at $815 \mathrm{~nm}$ as a function of polarization angle of input electric field calculated for $r=150 \mathrm{~nm}, p=590 \mathrm{~nm}$ and $h=440 \mathrm{~nm}$.

Figure 5(b) shows the calculated SH conversion efficiency for a FW of $815 \mathrm{~nm}$ as a function of the angle between the linearly polarized electric field of the pump and the y-axis (Fig. 1a). As seen, the SH conversion efficiency is only weakly polarization-sensitive. The maximum efficiency is obtained when the input polarization is set along the $y$-axis or the $x$-axis and it is minimum along the bisect between the $\mathrm{x}$ and $\mathrm{y}$-axes. However, the difference in conversion efficiency between these two extrema points is less than $20 \%$. This behavior of the SH conversion efficiency as a function of the incident polarization angle is due to the symmetry of the rectangular array 
and of the second-order susceptibility tensor, described by Eq. (2). In fact, the linear response of the metasurface (i.e. reflectivity and transmissivity) is polarization insensitive since the intensity of the excited multipoles (i.e. electric and magnetic dipoles) are constant as the polarization angle of the pump beam is varied.

We also performed numerical simulations for a configuration with the pump beam incident from the top of the metasurface (airside). In this scenario, we obtain very similar results for slightly different geometrical parameters. The optimal metasurface has pillar radius, pitch, and height of $170 \mathrm{~nm}, 657 \mathrm{~nm}$, and $680 \mathrm{~nm}$, respectively. The difference in geometry with respect to the case of the pump from the substrate side that we analyzed in the first place can be attributed to a different electric field localization of the FW beam in the two configurations (excitation from the air or the substrate side). Furthermore, since the structure is not symmetric along the z-axis, the impedance mismatch of the air-metasurface and the substrate-metasurface interfaces are different, which rescales the total intensity at the FW inside the metasurface. A more extensive analysis of this behavior will be the subject of future work.

\section{Conclusions}

In conclusion, we have proposed a new concept for nonlinear metasurface of monolithic material for enhanced SHG. We have demonstrated this concept in a monolithic $\mathrm{LiNbO}_{3}$ metasurface of periodic cylindrical nanopillar on $\mathrm{LiNbO}_{3}$ substrate. Using full-vectorial numerical simulations we observed a strong magnetic dipole resonance in the monolithic $\mathrm{LiNbO}_{3}$ nanoantennas, allowing for an average E-field intensity enhancement of over ten times. Such field intensity enhancement is comparable to that of the nanoantennas in air or on a low-refractive-index substrate. By finely adjusting size parameters on three dimensions, we have obtained an optimized SHG efficiency of over $5 \times 10^{-5}$ assuming a $1 \mathrm{GW} / \mathrm{cm}^{2}$ pump intensity. We have further analyzed the SHG efficiency modulation related to pillar radius and input polarization. Our results open the way for the development of nonlinear metasurfaces in transparent ferroelectric materials.

\section{Funding}

Australian Research Council (DP190101559); Foundation for Innovative Research Groups of the National Natural Science Foundation of China (11674167, 91850204); Deutsche Forschungsgemeinschaft (STA 1426/2-1); National Key Research and Development Program of China Stem Cell and Translational Research (2017YFA0303701); Università degli Studi di Padova (STARS-StG-PULSAr).

\section{Acknowledgments}

The authors acknowledge useful discussions with M. Celebrano and F. Setzpfandt.

\section{Disclosures}

The authors declare no conflicts of interest.

\section{References}

1. R. W. Boyd, Nonlinear Optics, (Elsevier, 2003).

2. C. Wang, X. Xiong, N. Andrade, V. Venkataraman, X.-F. Ren, G.-C. Guo, and M. Lončar, "Second harmonic generation in nano-structured thin-film lithium niobate waveguides," Opt. Express 25(6), 6963-6973 (2017).

3. L. Chang, Y. Li, N. Volet, L. Wang, J. Peters, and J. E. Bowers, "Thin film wavelength converters for photonic integrated circuits," Optica 3(5), 531-535 (2016).

4. C. Wang, Z. Li, M.-H. Kim, X. Xiong, X. Ren, G.-C. Guo, N. Yu, and M. Lončar, "Metasurface-assisted phase-matching-free second harmonic generation in lithium niobate waveguides," Nat. Commun. 8(1), 2098 (2017).

5. D. Smirnova and Y. S. Kivshar, "Multipolar nonlinear nanophotonics," Optica 3(11), 1241-1255 (2016). 
6. L. Carletti, D. Rocco, A. Locatelli, C. De Angelis, V. F. Gili, M. Ravaro, I. Favero, G. Leo, M. Finazzi, L. Ghirardini, M. Celebrano, G. Marino, and A. V. Zayats, "Controlling Second-harmonic Generation at the Nanoscale with Monolithic AlGaAs-on-AlOx Antennas," Nanotechnology 28(11), 114005 (2017).

7. A. I. Kuznetsov, A. E. Miroshnichenko, M. L. Brongersma, Y. S. Kivshar, and B. Luk'yanchuk, "Optically Resonant Dielectric Nanostructures," Science 354(6314), aag2472 (2016).

8. M. Kauranen and A. V. Zayats, "Nonlinear plasmonics," Nat. Photonics 6(11), 737-748 (2012).

9. R. Camacho-Morales, M. Rahmani, S. Kruk, L. Wang, L. Xu, D. A. Smirnova, A. S. Solntsev, A. Miroshnichenko, H. H. Tan, F. Karouta, S. Naureen, K. Vora, L. Carletti, C. De Angelis, C. Jagadish, Y. S. Kivshar, and D. N. Neshev, "Nonlinear Generation of Vector Beams From AlGaAs Nanoantennas," Nano Lett. 16(11), 7191-7197 (2016).

10. L. Carletti, A. Locatelli, D. N. Neshev, and C. De Angelis, "Shaping the second harmonic radiation pattern from AlGaAs dielectric nanoantennas," ACS Photonics 3(8), 1500-1507 (2016).

11. V. F. Gili, L. Carletti, A. Locatelli, D. Rocco, M. Finazzi, L. Ghirardini, I. Favero, C. Gomez, A. Lemaître, M. Celebrano, C. De Angelis, and G. Leo, "Monolithic AlGaAs second-harmonic nanoantennas," Opt. Express 24(14), 15965-15971 (2016).

12. L. Carletti, A. Locatelli, O. Stepanenko, G. Leo, and C. De Angelis, "Enhanced second-harmonic generation from magnetic resonance in AlGaAs nanoantennas," Opt. Express 23(20), 26544-26550 (2015).

13. S. Liu, M. B. Sinclair, S. Saravi, G. A. Keeler, Y. Yang, J. Reno, G. M. Peake, F. Setzpfandt, I. Staude, T. Pertsh, and I. Brener, "Resonantly Enhanced Second-Harmonic Generation Using III-V Semiconductor All-Dielectric Metasurfaces," Nano Lett. 16(9), 5426-5432 (2016).

14. P. P. Vabishchevich, S. Liu, M. B. Sinclair, G. A. Keeler, G. M. Peake, and I. Brener, "Enhanced Second-Harmonic Generation Using Broken Symmetry III-V Semiconductor Fano Metasurfaces," ACS Photonics 5(5), 1685-1690 (2018).

15. F. J. F. Löchner, A. N. Fedotova, S. Liu, G. A. Keeler, G. M. Peake, S. Saravi, M. R. Shcherbakov, S. Burger, A. A. Fedyanin, I. Brener, T. Pertsch, F. Setzpfandt, and I. Staude, "Polarization-Dependent Second Harmonic Diffraction from Resonant GaAs Metasurfaces," ACS Photonics 5(5), 1786-1793 (2018).

16. S. Buckley, M. Radulaski, K. Biermann, and J. Vučković, "Second harmonic generation in photonic crystal cavities in (111)-oriented GaAs," Appl. Phys. Lett. 103(21), 211117 (2013).

17. J. D. Sautter, L. Xu, A. E. Miroshnichenko, M. Lysevych, I. Volkovskaya, D. A. Smirnova, R. Camacho-Morales, K. Z. Kamali, F. Karouta, K. Vora, H. H. Tan, M. Kauranen, I. Staude, C. Jagadish, D. N. Neshev, and M. Rahmani, "Tailoring Second-Harmonic Emission from (111)-GaAs Nanoantennas," Nano Lett. 19(6), 3905-3911 (2019).

18. A. Sergeyev, R. Geiss, A. S. Solntsev, A. Steinbrück, F. Schrempel, E.-B. Kley, T. Pertsch, and R. Grange, "Second-harmonic generation in lithium niobate nanowires for local fluorescence excitation," Opt. Express 21(16), 19012-19021 (2013).

19. C. Wang, M. Zhang, B. Stern, M. Lipson, and M. Lončar, "Nanophotonic lithium niobate electro-optic modulators," Opt. Express 26(2), 1547-1555 (2018).

20. D. Lehr, J. Reinhold, I. Thiele, H. Hartung, K. Dietrich, C. Menzel, T. Pertsch, E.-B. Kley, and A. Tünnermann, "Enhancing Second Harmonic Generation in Gold Nanoring Resonators Filled with Lithium Niobate," Nano Lett. 15(2), 1025-1030 (2015).

21. B. Gao, M. Ren, W. Wu, H. Hu, W. Cai, and J. Xu, "Lithium Niobate Metasurfaces," Laser Photonics Rev. 13(5), 1800312 (2019).

22. F. Timpu, J. Sendra, C. Renaut, L. Lang, M. Timofeeva, M. T. Buscaglia, V. Buscaglia, and R. Grange, "Lithium Niobate Nanocubes as Linear and Nonlinear Ultraviolet Mie Resonators," ACS Photonics 6(2), 545-552 (2019).

23. A. Fedotova, M. Younes, J. Sautter, R. Geiss, T. Pertch, I. Staude, and F. Setzpfandt, "Second-Harmonic Generation in Lithium Niobate Metasurfaces," in CLEO/Europe-EQEC 2019, Munich, 2019.

24. S. Goeman, S. Boons, B. Dhoedt, K. Vandeputte, K. Caekebeke, P. Van Daele, and R. Baets, "First demonstration of highly reflective and highly polarization selective diffraction gratings (GIRO-gratings) for long-wavelength VCSEL's," IEEE Photonics Technol. Lett. 10(9), 1205-1207 (1998).

25. M. Gębski, M. Dems, A. Szerling, M. Motyka, L. Marona, R. Kruszka, D. Urbańczyk, M. Walczakowski, N. Pałka, A. Wójcik-Jedlińska, Q. J. Wang, D. H. Zhang, M. Bugajski, M. Wasiak, and T. Czyszanowski, "Monolithic high-index contrast grating: a material independent high-reflectance VCSEL mirror," Opt. Express 23(9), 11674-11686 (2015)

26. M. Marciniak, M. Gębski, M. Dems, E. Haglund, A. Larsson, M. Riaziat, J. A. Lott, and T. Czyszanowski, "Optimal parameters of monolithic high-contrast grating mirrors," Opt. Lett. 41(15), 3495-3498 (2016).

27. P. Spinelli, M. Verschuuren, and A. Polman, "Broadband omnidirectional antireflection coating based on subwavelength surface Mie resonators," Nat. Commun. 3(1), 692 (2012).

28. J. Cambiasso, G. Grinblat, Y. Li, A. Rakovich, E. Cortés, and S. A. Maier, "Bridging the Gap between Dielectric Nanophotonics and the Visible Regime with Effectively Lossless Gallium Phosphide Antennas," Nano Lett. 17(2), 1219-1225 (2017).

29. D. E. Zelmon, D. L. Small, and D. Jundt, "Infrared corrected Sellmeier coefficients for congruently grown lithium niobate and 5 mol. \% magnesium oxide-doped lithium niobate," J. Opt. Soc. Am. B 14(12), 3319-3322 (1997).

30. D. Yudistira, A. Boes, B. Graczykowski, F. Alzina, L. Y. Yeo, C. M. S. Torres, and A. Mitchell, "Nanoscale pillar hypersonic surface phononic crystals,” Phys. Rev. B 94(9), 094304 (2016).

31. R. Alaee, C. Rockstuhl, and I. Fernandez-Corbaton, "An electromagnetic multipole expansion beyond the longwavelength approximation," Opt. Commun. 407, 17-21 (2018). 